\title{
Multi-agent systems to improve efficiency in steelworks ${ }^{\text {is }}$
}

\author{
Vincenzo Iannino ${ }^{1, *}$ (D), Valentina Colla ${ }^{1}$ (D), Claudio Mocci ${ }^{1}$, Ismael Matino ${ }^{1}$, Stefano Dettori ${ }^{1}\left(\mathbb{D}\right.$, Sebastian Kolb ${ }^{2}$ (D) \\ Thomas Plankenbühler², and Jürgen $\mathrm{Karl}^{2}$ \\ ${ }^{1}$ Scuola Superiore Sant'Anna, TeCIP Institute, ICT-COISP Center, Via Moruzzi 1, 56124 Pisa, Italy \\ ${ }^{2}$ Friedrich-Alexander Universität Erlangen-Nürnberg (FAU), Chair of Energy Process Engineering, Fürther Str. $244 f, 90429$ \\ Nürnberg, Germany
}

Received: 11 September 2021 / Accepted: 27 January 2022

\begin{abstract}
A multi-agent system consists of several computational entities capable of autonomous actions, called agents, which communicate with each other, and have the ability to coordinate their actions and to cooperate. Multi-agent systems received a great interest and attention over time, as they can be seen as a key enabling technology for complex applications, where distributed and processing of data, autonomy, and high degree of interactions in dynamic environments are required at the same time. Therefore, in view of current and future developments of the digitalization of industrial production cycles promoted by Industry 4.0, multi-agent systems are foreseen to play an increasing role for industrial production management and optimization. Because of barriers represented by large presence of legacy systems, in the steel sector agent-based technology is not widely applied yet, and multi-agent systems applications are very few. On the other hand, steel manufacturing industries are complex and dynamic systems whose production processes held a strategic role in the global economy. During last decades, the steel sector has undergone relevant transformations, especially through the massive digitalization and the innovation introduced by Industry 4.0. A further evolution is foreseen in the incoming years to improve the sustainability of the production cycle by improving energy and resource efficiency. Therefore, steel industries must face several challenges on the path toward the factory of the future. In such context multi-agent systems, through their intrinsic properties, such as autonomy, social abilities, reactivity, proactivity, and mobility, can overcome existing drawbacks and barriers, by increasing flexibility, improving resources efficiency, handling production operations, reacting to unpredicted events, optimizing production processes, and supporting legacy systems. In this paper, some applications of multi-agent systems in steel sector are presented to show the advantages and opportunities of agent-based technology.
\end{abstract}

Keywords: Industry 4.0 / Multi-Agent Systems / dynamic resources allocation / off-gas management / steel industry / sustainability

\section{Introduction}

Steel manufacturing industries are large, complex, and dynamic systems whose production processes held a strategic role in the global economy. According to European Steel Association report [1], the European steel industry is world leader in innovation and environmental sustainability: it creates around $€ 140$ billion of Gross Value Added (GVA); the sector produces on average 170 million tonnes of finished steel per year; the European steel industry has 500 production sites spread out across 23 EU Member States, employs 330000 people directly, and supports nearly 2.6 million jobs.

\footnotetext{
it 15th International Conference on Society \& Materials, SAMEcoSD-15, Virtual, 10-11 May 2021.

* e-mail: valentina.colla@santannapisa.it
}

The massive digitalization and the innovation introduced by Industry 4.0 (I4.0) is pushing process manufacturing industries towards a new vision of production systems, the so-called Smart Factories, which include important features, such as agile and flexible production structures, vertical and horizontal integration of production systems through Information and Communication Technology (ICT) systems, interoperability and real-time capability of fast reaction to changing process conditions and product requirements [2]. In this scenario, the steel sector is experiencing this new transformation, which opens new possibilities for implementing advanced approaches, including Cyber-Physical Systems (CPS), Industrial Internet of Things (IIoT), and Artificial Intelligence (AI)-based concepts. The application of new technologies can really support the optimization of the entire production cycle. The intelligent combination of process automation, 
Information Technology (IT)-based systems and connectivity enable the digitalization of steel production that goes far beyond the conventional automation of industrial production. Nevertheless, European Steel Industries is facing several challenges on the path toward the I4.0 paradigm [3]. Some of them are the following ones:

- to enable a smooth and economic viable transition towards I4.0 technologies, e.g., through gradual migration from the monolithic ISA95/IEC62624 pyramidal model [4] to more suitable architectures, such as, for instance, RAMI4.0 [5];

- to efficiently manage energy sources and energy carriers, e.g., process gases, to increase the economic and environmental sustainability of the integrated steelworks production by reducing $\mathrm{CO}_{2}$ emissions [6];

- to improve the current process logistics and the adopted optimization solutions to reduce energy consumptions and loss of efficiency in the production;

- to improve the allocation and efficient exploitation of plant resources to maximize the productivity by reducing waste of materials and production costs [7];

- to handle unexpected events within the process by promptly reacting to them and mitigating their effects [8];

- to enhance communication among subprocesses;

- to support common IT infrastructures and legacy systems.

But how to face steelworks challenges in the I4.0 era? Multi-Agent Systems (MASs) represent a suitable approach to address the new generation of flexible, digitalized, intelligent and distributed manufacturing processes $[9,10]$, especially steel manufacturing industries, which require the consideration of several different constraints and objectives of a class of processes. Agent-based approaches play a major role as a fundamental technology for industrial production processes and resources management and optimization [11], as they provide efficient and interesting solutions to a wide range of problems in different areas of interest. The potential advantages in using agent-based technologies are numerous and relevant in all domains of manufacturing [12]. However, the applications of MASs in the steel sector are still limited compared to their potentials and related benefits. Some noticeable examples of MAS practical applications can be found related to process scheduling [13,14], dynamic product reallocation [15] and dynamic management of energy consumptions in electric steelworks $[16,17]$.

This paper reports two recent exemplar applications of MASs in steel sector, which show the advantages and opportunities of agent-based technology to improve process efficiency as well as valorisation of an important by-product, namely process off-gases, by also leading to emissions reduction. These two examples show how MASs can be beneficial to improve the sustainability of steel production processes from both economic and environmental points of view.

In this paper, Section 2 briefly reports some background about MASs. Section 3 presents two exemplar applications of MAS in steelworks. More in detail, the former is focused on the improvement of production scheduling flexibility in flat steel production through intelligent dynamic resource allocation. The second application concern the increase of economic and environmental sustainability of steelworks by off-gases' management and valorisation through hydrogen enrichment aimed at methane and methanol synthesis. Section 4 discusses the main barriers and challenges towards the application of agent-based technology in the steel sector. Finally, Section 5 provides some concluding remarks and hints for future developments.

\section{Background on multi-agent systems}

Knowledge about MASs dates back in eighties within the field of Distributed Artificial Intelligence (DAI). DAI investigates the design of distributed, interacting systems and associated issues. It involves three main topics that are: Parallel Artificial Intelligence (PAI), Distributed Problem Solving (DPS) and MASs. PAI investigates the extent to which complex problem solutions can be accelerated by their distribution to a larger number of resources (parallel hardware and software processes). DPS investigates the extent to which complex problems can be solved by the assignment to modules that cooperate with each other by exchanging their knowledge. On the other hand, MASs involve the study of the behaviour of multiple interacting entities called agents.

During the past decades, various definitions of agent have been proposed in literature [18], and what emerged from that is that an agent is a computational entity which exhibit some characteristics such as autonomy, reactiveness, proactiveness, social ability, learning capabilities and mobility. In a MAS, existing agents are extended with communication and interaction capabilities, e.g., coordination, negotiation, responsibility delegation, and they interact to solve problems that are beyond their individual capabilities or knowledges. Agents' communication is usually realized through direct exchanging messages among them according to a communication language. The most important agent communication languages are the Knowledge Query and Manipulation Language (KQML) developed by DARPA and the Agent Communication Language (ACL) standardized by the Foundation for Intelligent Physical Agents (FIPA) [19], but simplified languages are also adopted. On the other hand, interaction is based on various coordination and cooperation approaches [20,21] ranging from coordination protocols, e.g., the well-known Contract Net Protocol (CNP), to distributed multi-agent planning, e.g., the Partial Global Planning (PGP). A brief list with the main characteristics of MASs is reported in Table 1.

MASs have received increasing attention not only in academia but also in industry, due to their applicability and versatility in solving complex problems. In infrastructure and energy domains, a survey of agent applications for the improvement of building operations and for control and operation of smart grids was presented in [22] and [23], while a survey of agent applications in different manufacturing domain was presented in [12]. MASs are used in the transportation sector for dynamic routing, congestion management, and intelligent traffic control [24], but also in healthcare, in particular, for realizing daily living and monitoring as well as telemedicine [25]. 
Table 1. Characteristics of agent-based systems.

Decentralization

Coordination and cooperation

Scalability and flexibility

Adaptability

Computational Efficiency

Robustness

Leanness
Agents are distributed and they communicate with each other exchanging messages over a network.

Agents can interact with each other and coordinate their actions in order to satisfy their design objectives.

MASs being distributed and loosely coupled computing systems can be adapted according to the problem size and agents can be replaced by other agents or new ones may enter the system.

Agents in a MAS are able to adapt their tasks and to react to changing environment conditions.

Agents can operate asynchronously and in parallel, solving sub-tasks separately and exploiting parallel computation. Thus, they provide high efficiency and increased overall speed.

MASs provide a high level of robustness and fault-tolerance, as consequence the failure of one or more agents does not necessarily make the overall system useless.

Agents in a MAS should be simples with few functionalities avoid complex behaviours. In this way agents are less expensive than a complex agent and easier to maintain.

\section{Two exemplar applications of multi-agent systems in steel production}

Over the last few decades, some steps forward have been done in the European steel industries through some projects funded by the EU through the Research Fund for Coal and Steel (RFCS) as well as through different initiatives related to $\mathrm{I} 4.0$ and digitalization developed under the umbrella of the European Steel Technology Platform (ESTEP). These projects/initiatives can be considered as good indicators of the importance of new technologies to innovate and improve the competitiveness of European steel companies. In the context of MASs, the very first project targeting the implementation of a MAS in steelworks was funded by the RFCS and was entitled "Development of a new automation and information paradigm for integrated intelligent manufacturing in steel industry based on holonic agent technology" (I2MSteel). In this project, a platform of agents and holons was developed to perform high level tasks, such as product tracking, process planning, through-process quality control, information storage and logistics [15]. A further RFCS project entitled "Economic and flexible decentral self-optimising production (SOPROD)" focused on the development of a distributed agent-based system platform aimed at increasing flexibility, resource efficiency and productivity as well as decreasing energy consumption of the steel production. This project inspired further projects such as the RFCS project Cyber-POS (Virtual Design of Cyber-Physical Production Optimization Systems for Long Production Factories), which was focused on establishing the feasibility, safety, and benefits of Cyber-Physical Production Systems (CPPS) through different software technologies including agents [26]. Moreover, in the project MAGUS
(Manufacturing Execution System Augmented Solution) funded by Regione Piemonte (Italy) and EU, where a MAS was developed to the aim of providing a technological solution to the specific needs of the process industry [27].

In the following subsections, two more recent examples of application of MAS in the context of the steel industry are presented, which concern dynamic allocation of resources to enhance process flexibility. In the first case, resources are machines and cranes, while in the second case, they are gaseous streams to be exploited for power production or synthesis of methane and methanol.

\subsection{Dynamic resource allocation for a flexible production scheduling in flat steel production}

Different types of steel products are manufactured in steelworks: flat steel products, such as steel sheets or steel plates, used in a broad range of applications across all industrial sectors and application in daily life. In this context, management and allocation of plant resources represent crucial aspects since the productivity quality of final products depend on robust production scheduling strategies. Production planning and scheduling determine the processing times and working sequences for each production order to satisfy manufacturing constraints, delivery dates and required qualities. The study of scheduling in steel plants has been one of the most important issues in the last decades becoming very popular in the scientific and technological literature [28,29]. Since most steel manufacturing plants operate in complex dynamic environment, where unpredictable events of different nature occur, e.g., machines failures, urgent jobs arrivals or quality problems, a flexible scheduling strategy and supporting tools must be available to cope with these issues. 


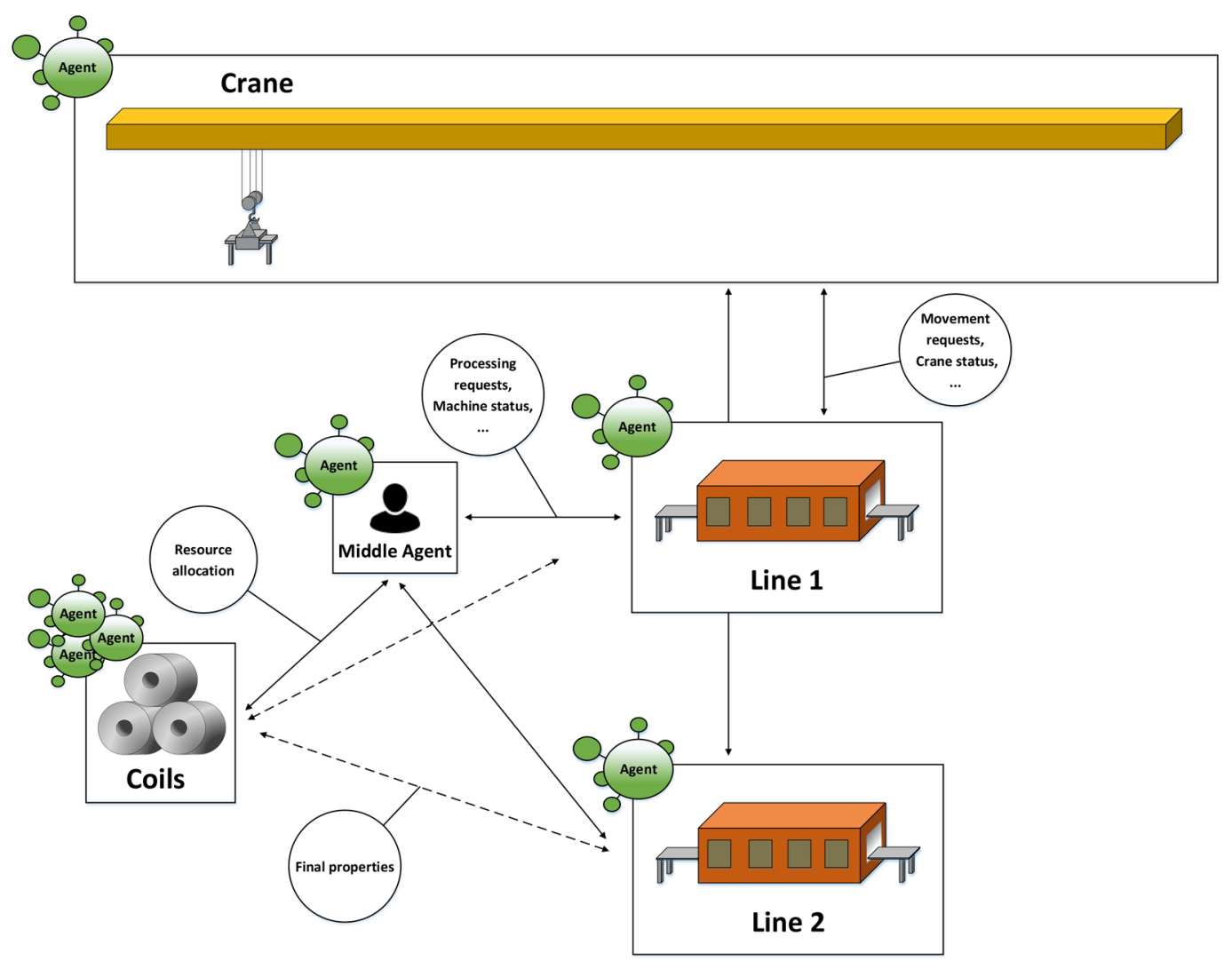

Fig. 1. Agent-based model for dynamic resource allocation.

In cold rolling and finishing area for flat steel production system, hot-rolled coils are transformed into flat products of different size and quality. Four main production steps are typically carried out in this area: pickling, cold rolling, annealing and temper or skin-pass rolling [30]. Assuming that each step is composed by two parallel lines, steel coils move from one stock to a production line by means of crane. At the end of one production step, semi-finished coils are stocked waiting to be process at the following step. The main objective is to optimize the overall plant utilization covering machine breakdowns and special maintenance operations that can compromise the normal production flow.

To solve this issue, agent-based systems intervene providing a flexible production scheduling considering the dynamic environment $[9,31]$ thanks to their intrinsic properties (see the above Tab. 1), and in particular, their ability of coordination and cooperation. Here an example is proposed, showing how agents can deal with the problem of the dynamic resource allocation of the considered flat steel production system. A MAS has been designed, which comprises four main agents at each production step as shown in Figure 1.

The following agents types are available:

- Coil Agent (CoA): it handles the routing that its strip follows inside the production plant;

- Crane Agent (CrA): it manages the transportation of coils within the assigned production step;
- Machine Agent (MachA): it implements a smart production line and represents a resource usable by coils; - Middle Agent (MdleA): it coordinates the resources allocation by centralizing the demands of CoAs.

Specifically, CoAs send resource allocation requests to the MdleA to be processed by one machine at the current production step. In order to facilitate and manage the communication and the resources allocation among CoAs and MachAs (Line 1 and Line 2 in Fig. 1), a MdleA for each production step is set up. The resource allocation is carried out by means of a customized brokering-based interaction protocol [32], where the MdleA takes the role of a broker, which receives requests messages from CoAs, and finds suitable machines able to satisfy CoAs requests. Then, the MdleA forwards those messages to MachAs, waits for their response, and sends the results back to CoAs. Each machine is represented by an independent agent, which communicates with the MdleA, the CrA and the CoAs. A machine is a resource that the MdleA allocates to a product one a time. When a machine resource is allocated to a product, the corresponding MachA contacts the CrA asking to bring the assigned product to its input buffer to be processed. Each crane is managed by an agent, which is in charge to transport products from the input stock to the input machines buffer. Due to highly dynamic production characterizing steel processes, unexpected events, such as special 
maintenance operations or breakdowns, can alter the normal production flow and affect both machines and cranes. In order to handle these unforeseen events, MachAs and CrAs are designed to send special messages warning the agents involved in the current production step.

The designed MAS was developed through Microsoft. Net@ cross-platform and C\# Objective Oriented Programming (OOP) language, and it was deployed over an hybrid peer-to-peer $(\mathrm{P} 2 \mathrm{P})$ distributed system [33], where each node is an intelligent and collaborative agent. Agents are represented by peers, which run concurrently in parallel, and are designed and implemented as multithreading entities. Once an agent is created and initialized, it is able to retrieve peers information according to its behaviour, and to exchange asynchronous messages with other agents to coordinate their actions. For this purpose, a central Server is used, which keeps tracks of every peer that is connected to the industrial network, and helps peers to find each other. Furthermore, the Server is used to get and update process data as well. This kind of architecture is easy to manage and to deploy on common Brownfield scenarios, where traditional IT infrastructures are adopted.

An example of results of the dynamic plant resources allocation is shown in the Gantt charts depicted in Figure 2. The production of fifteen products has been simulated in undisturbed conditions (Fig. 2a) and with perturbations affecting the production system (Fig. 2b). Specifically, different unexpected events have been randomly generated on-line during the simulation and are highlighted in red (MAINT/BREAK).

In deeper details, a breakdown affects one of the pickling lines at stage one (P2), the crane at stage 3 (Crane3) is temporary suspended by a maintenance operation, a further maintenance operation causes a suspension of the activity of both the cold rolling line at stage two (CR2) and the continuous annealing line at stage three (CA2). Despite the perturbed conditions, a feasible scheduling is provided by agents, in particular, MdleAs are able to coordinate the resources allocation process, and the residual global Makespan computed as the difference of the completion time of the last coil between the first and the second scenario is about 4 minutes over $3 \mathrm{~h}, 37$ minutes and $49 \mathrm{~s}$ of production, representing a production shift of $1.83 \%$ without capacity losses which usually happens within disturbed scenarios [34,35]. The overall production flow is not compromised, showing a good flexibility and utilization of the plant resources through the designed agent-based system.

\subsection{Off-gases management and valorisation through hydrogen enrichment for methane and methanol synthesis}

During primary steel production within an integrated steel plant, three different types of process off-gases are produced, i.e., Coke Oven Gas (COG), Blast Furnace Gas (BFG) and Basic Oxygen Furnace Gas (BOFG), which are, respectively, rich in hydrogen $\left(\mathrm{H}_{2}\right)$, carbon dioxide $\left(\mathrm{CO}_{2}\right)$ and carbon monoxide $(\mathrm{CO})$. These gases are

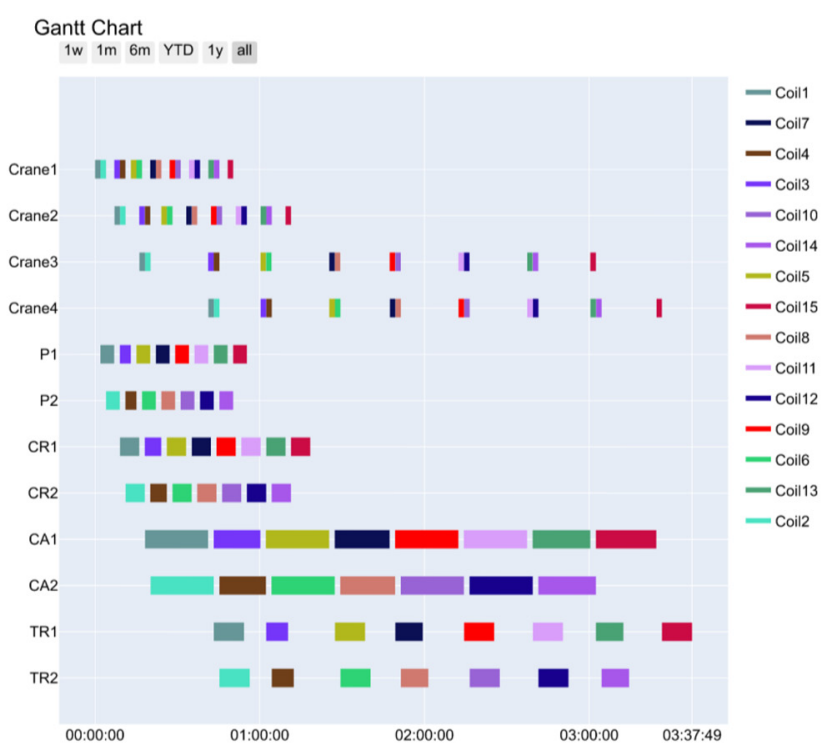

(a)

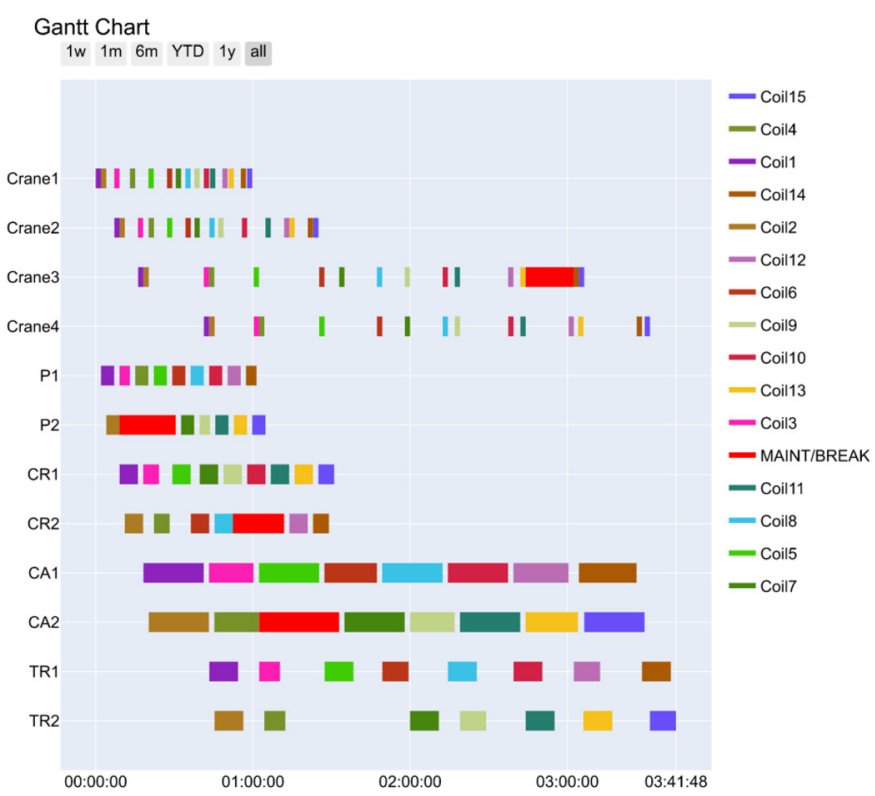

(b)

Fig. 2. Dynamic plant resources allocation in flat steel manufacturing through MASs: (a) without unexpected events; (b) with unexpected events.

usually exploited to meet the energy demand of production processes, to produce steam and as energy sources for power plants, which can both satisfy internal electricity demand and sell part of their production on the external energy market. An alternative way of utilizing off-gases is to exploit chemical synthesis reactors converting the carbon content to more valuable products, such as methane $\left(\mathrm{CH}_{4}\right)$ [36] and methanol $\left(\mathrm{CH}_{3} \mathrm{OH}\right)$, through the enrichment of by-product gases with $\mathrm{H}_{2}$. The project entitled "Integrated and intelligent upgrade of carbon sources through hydrogen addition for the steel industry" (i3upgrade), which is funded by Research Fund for Coal 


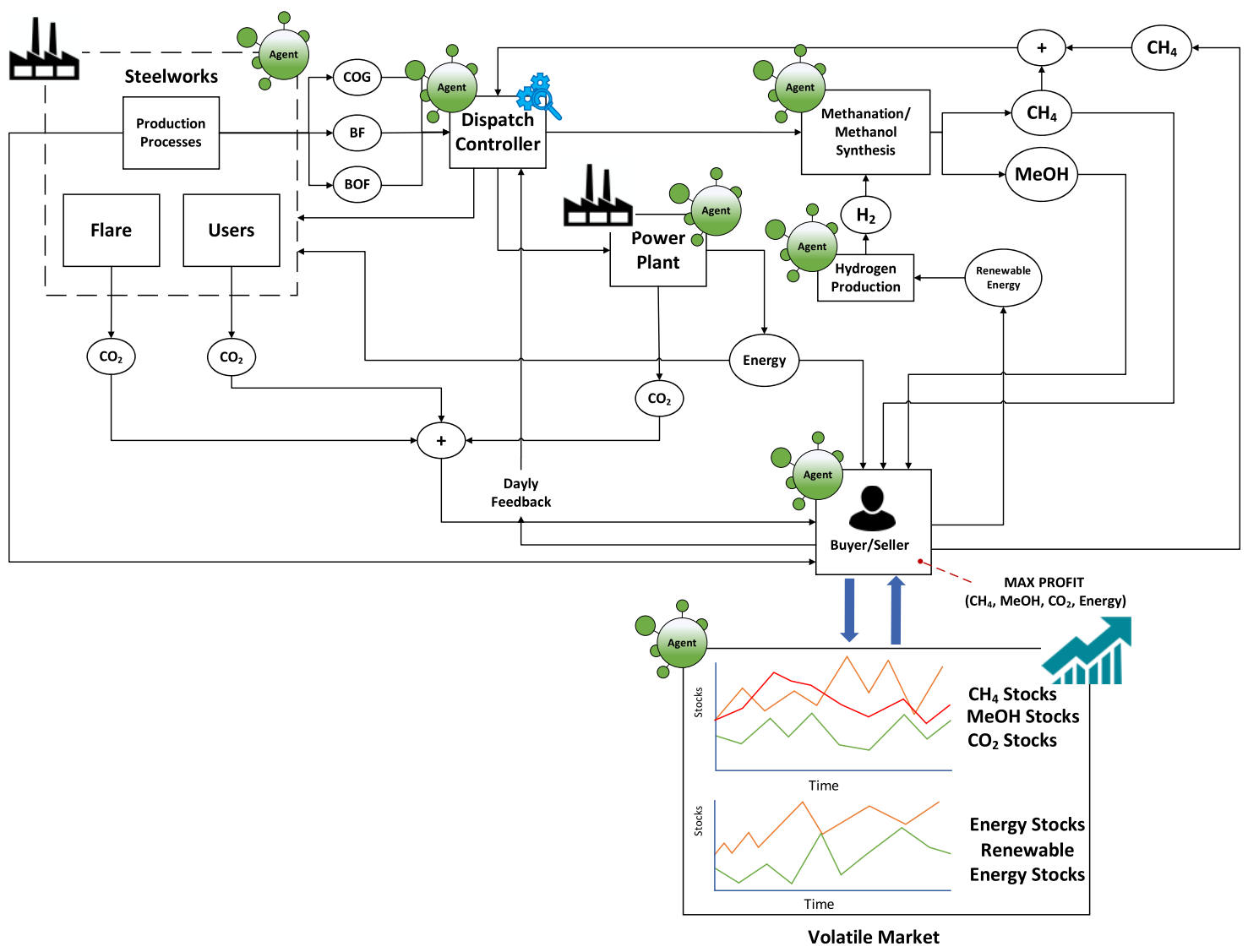

Fig. 3. Designed MAS model with main interactions among agents.

and Steel (RFCS) of European Union (EU), aims at efficiently managing process off-gases to increase the economic and environmental sustainability of the integrated steelworks production. Through the addition of H2 produced by electrolysis powered by renewable sources it is possible to reduce $\mathrm{CO}_{2}$ emissions by improving the sustainability of the steel production cycle $[37,38]$. Another aspect covered by i3upgrade is the evaluation of long-term economic profitability of hydrogen-intensified syntheses in integrated steelworks as well as the derivation of potential business models and implementation strategies for the European steel industry and beyond. In order to cope with these tasks, an agent-based system model is implemented to investigate how optimizing off-gases utilization, to reduce the $\mathrm{CO}_{2}$ emissions, and by minimizing Operating Expense (OPEX) and Capital Expenditure (CAPEX) costs in the emerging volatile markets.

The agent-based model is shown in Figure 3. The model comprises all relevant parts of the steelworks with detailed reactor models, the dynamic representations of markets allowing for the evaluation of future economic and environmental benefits of the proposed concepts.

The MAS is composed by the following main agents:

- Steelworks Agents (SAs): they are responsible for providing the global amount and the main features of offgases;
- Power Plant Agent (PPA): it aims at producing electric energy by using part of the available steelworks gases. Steelworks gases could be indeed used both for internal steelworks users, in power plant or in the synthesis reactors. The produced electricity is, therefore, used to supply the processes in the steelworks and the surplus of energy could be used to maximize the profit of the integrated steelworks by selling it on the market;

- Reactors Agents (RAs): they represent the methanation and methanol syntheses as well as the hydrogen production units;

- Buyer/Seller Agent (BSA): it optimises the operation of the hydrogen intensified synthesis options in the integrated steelworks from an economic point of view and sends daily feedbacks to the dispatch controller. The BSA has knowledge of the time-resolved requirements of the steelworks (in terms of its natural gas demand), the developments of the volatile markets and abstracted information about the Key Performance Indicators (KPIs) of the single reactors. The agent defines hourly production targets for the synthesis options for the upcoming $24 \mathrm{~h}$;

- Dispatch Controller Agent (DCA): it optimizes the distribution of process off-gasses and controls the operation modes of the reactors and power plant. It translates the economically optimized production targets of the BSA into detailed instructions for RAs and PPA; 
- Market Agent (MkA): it delivers the relevant commodity prices for electricity, natural gas (NG), $\mathrm{CO}_{2}$ and $\mathrm{CH}_{3} \mathrm{OH}$ to the $\mathrm{BSA}$ based on the volatile market dynamic. The dynamic representation of the $\mathrm{MkA}$ is crucial to allow the investigation of different future market conditions. Price and volatility predictions serve as basis for the adaptation of the market models to future market situations.

The agent-based model has been simulated through the AnyLogic@ software tool, which supports three types of simulation modelling methods, i.e., system dynamics, discrete event, and agent based modelling, and allows creating multi-method models. The behaviour of each agent has been modelled through state charts, which are suited for describing event- and time-driven behaviours. The optimisation problems have been formulated through GUROBI@ optimizer, that is powerful mathematical optimization solver, which can be integrated within AnyLogic@.

The agent-based model allows the simulation of single (future) years or multiple consecutive years (e.g., scenarios until 2050). This makes it possible to investigate both the performance of the hydrogen intensified synthesis options under a given future market framework and their long-term profitability given time-dependent changes of market conditions. The model time step is set to $1 \mathrm{~h}$, which represents a trade-off among computational effort of the simulation, time resolution of the considered markets and relevant timescales of the reactors. Figure 4 shows a flow chart with the sequence of simulation steps of the different agents. On start-up, the model initialises the first day and hour of the year. In a first step, MkA predicts electricity, methanol, $\mathrm{CO}_{2}$ and NG prices for the upcoming $24 \mathrm{~h}$. This comprises electricity prices in hourly resolution (representing day-ahead spot market prices) and $\mathrm{NG}, \mathrm{CO}_{2}$ and methanol prices in daily resolution. Using the information of price developments, RAs and PPA calculate the costs for each of their possible states (e.g., start-up, ready, in operation) for the upcoming and preceding $24 \mathrm{~h}$. RAs and PPA combine system dynamics and discrete event methods to comprehensively model the economic, environmental and technical performance of the hydrogen intensified synthesis options. Subsequently, SAs forecast the steelworks parameters in terms of NG demand and availability of off-gases for the whole day. Since off-gases production is characterized by discontinuous dynamics in terms of both volume flow and composition, due to the variability of the scheduling of blast furnace, coke ovens and basic oxygen furnaces as well as to the features of involved processes, off-gases excess can be easily forecasted through data-driven methodologies including Artificial Intelligence (AI) techniques $[39,40]$. In the present application, Echo State Neural Networks (ESN) are used for this purpose [39]. The ESN integrated into SAs are used to predict off-gases production and then the results are sent by SAs to the DCA. Every $6 \mathrm{~h}$ (recurrence time) the BSA performs the economic optimisation of the power plant and reactors production targets for the next $24 \mathrm{~h}$ in a rolling optimisation. The optimization is performed through a
Mixed-Integer Linear Programming (MILP) approach where the cost function to optimize is computed as:

$$
\min \sum_{k=0}^{23}\left(J_{\text {market }}(k)+J_{\text {OPEX }}(k)-J_{\text {revenues }}(k)\right),
$$

where $J_{\text {revenues }}(k)$ are the revenues for selling methane and/ or methanol, and electricity generation, $J_{\text {market }}(k)$ are the market costs due to purchasing natural gas from the market, and $J_{O P E X}(k)$ are the operative costs for switching the equipment, i.e., electrolysis, reactors, and power plant, considering electricity and $\mathrm{CO}_{2}$ emissions costs as well. The main constraints of the optimization problem describe the fulfilment of the internal natural gas demand at each step and the balancing of hydrogen production from electrolysis and hydrogen consumption from reactors.

In doing the optimization, the BSA exploits the prices determined by the MkA, the costs from the RAs and PPA as well as their operating states in the beginning of the optimisation horizon. Using the optimised results of the BSA, the DCA triggers the operating states of the different RAs and PPA sending messages to them. Since the optimization performed by the BSA is focused on longterm, it is necessary to adopt a control strategy in the shortterm to make reactors, power plant and equipment operating in safe and non-discontinuous conditions, while reacting to disturbances typical of process off-gases network distribution in terms of discontinuous production and internal consumptions. For this purpose, the DCA implements a Model Predictive Control (MPC) approach [41] based on the solution of a MILP where the prediction/ control horizon is set to $2 \mathrm{~h}$. The cost function used for the optimization is the following:

$$
\min \sum_{k=0}^{1}\left(J_{O P E X}(k)-J_{\text {revenues }}(k)\right),
$$

where $J_{\text {revenues }}(k)$ are the revenues for selling methane and/ or methanol, and electricity generation, and $J_{O P E X}(k)$ are the operative costs that consider electricity consumption in the electrolyzer, $\mathrm{CO}_{2}$ emissions costs as well as costs related to equipment switching (i.e., electrolysis, reactors, power plant). The constraints included in the optimization problem describe the dynamics of each process involved, and the related operative limits. More in details, the limits involve the gasholder levels, the reactors behaviour (i.e., $\min / \max$ inlet gas volume flows, $\min / \max$ Stoichiometric Number, and modalities), the electrolyzer dynamics (i.e., $\min /$ max number of active stacks and min/max production of hydrogen), and the power plant (i.e., $\min / \max$ power of each group and $\min / \max$ load variation).

Finally, RAs and PPA thereupon change their states and perform the actions related to the respective change (e.g., heating up the reactor and calculating its temperature). Then, in an iterative loop, the simulation procedure restarts with the price predictions of the MkA in the next hour.

A preliminary set of significant results relevant to different simulations through the designed MAS is reported in Figures $5-7$. Figure 5 shows two hours ahead prediction 


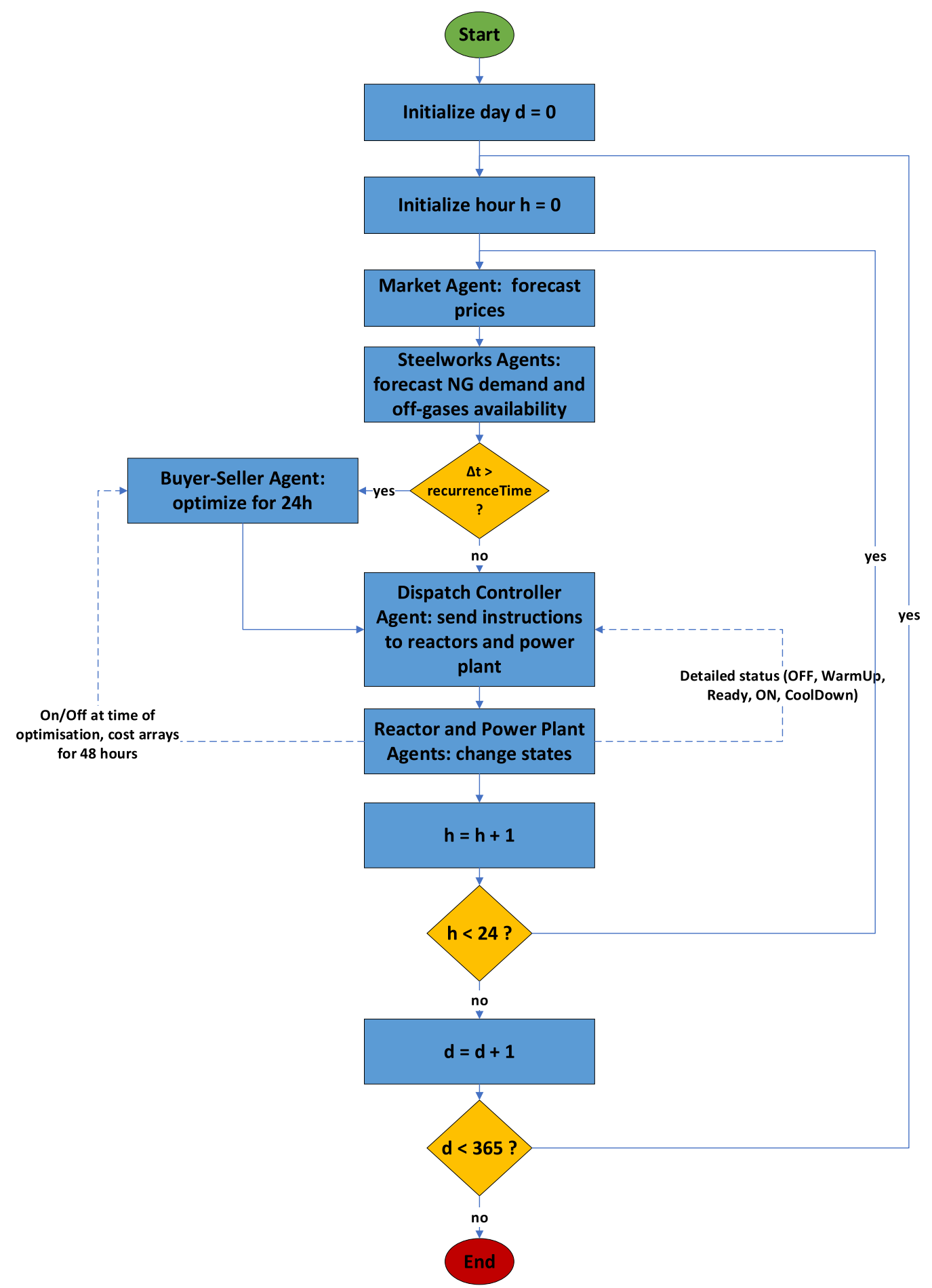

Fig. 4. Flow Diagram representing the sequence of simulation steps in the agent-based model.

of both BFG and BOFG volume provided by SAs, where the target is depicted in blue and the ESN forecasts in orange. Figure 6 graphically represents the operating principle of the DCA. It displays the feedback of the BSA (orange line) expressed by an on/off command, which suggests when turning on or off the reactors, and the methane synthesis reactor temperature (blue line) as a direct consequence of the decision-making process of the DCA. A temperature of $250^{\circ} \mathrm{C}$ represents operation of the reactor but also the ready state, in which the temperature is held constant to be ready for immediate operation. The DCA ensures that the reactor starts heating-up on time, according to suggestions of the BSA. In a similar way, the dispatch controller triggers the methane reactor's cooldown operation. Finally, Figure 7 shows the management of off-gases realized through the DCA. It is possible to see how gases are dispatched to reactors (orange lines) or to the power plant (blue lines) according to the results of the 


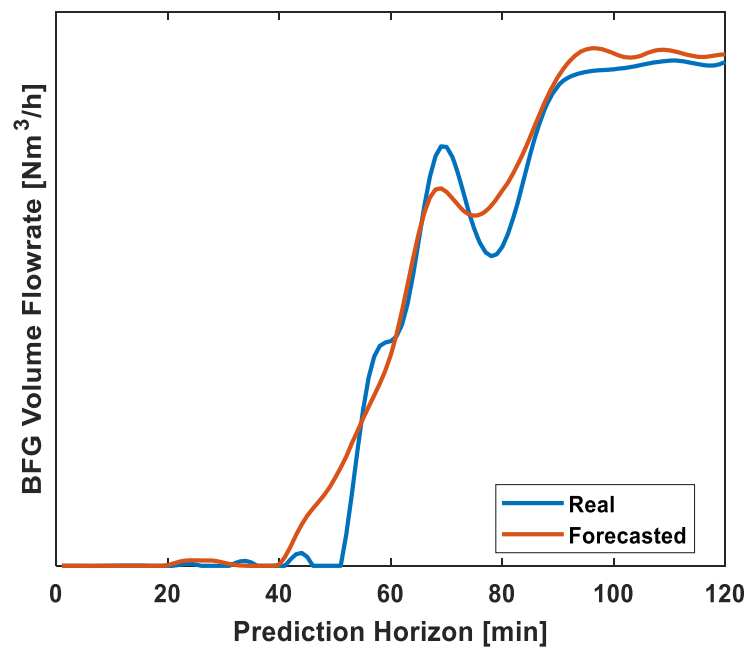

(a)

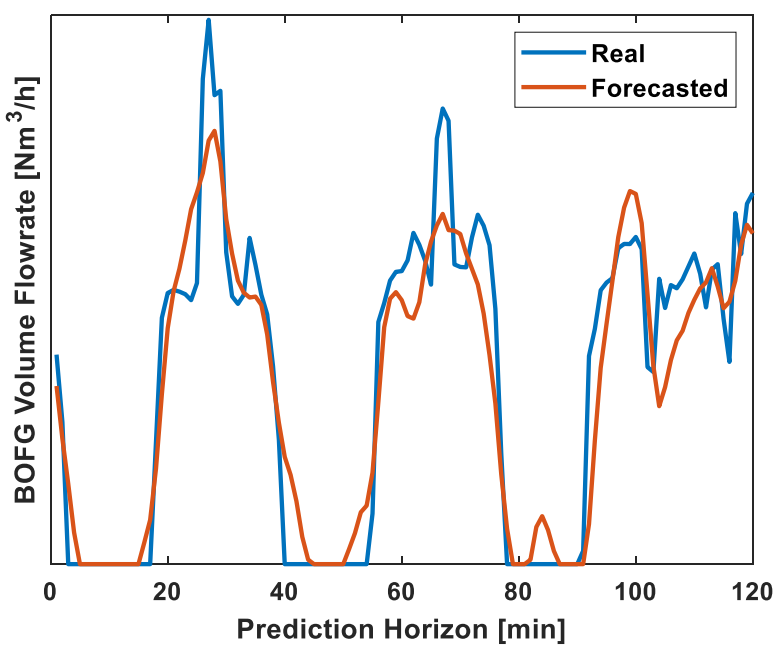

(b)

Fig. 5. Prediction example of off-gases volume flow production: (a) blast furnace gas flow rate; (b) basic oxygen furnace gas flow rate.

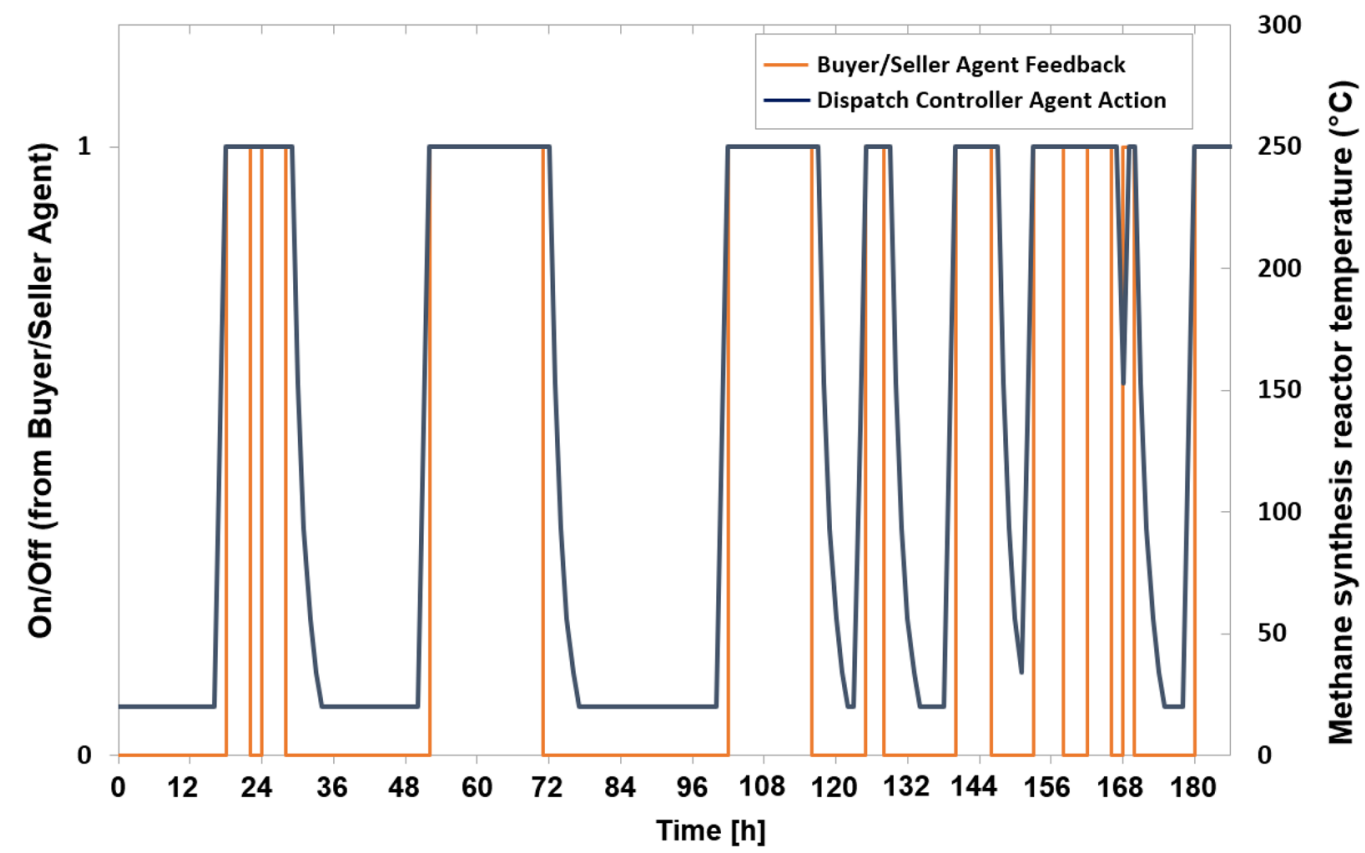

Fig. 6. Exemplary output of the dispatch controller agent: control of the methane synthesis reactor temperature status based on the operating decisions of the buyer/seller agent.

optimization and the feedbacks of the buyer/seller agent. The examples already presented show only preliminary results since the work is still ongoing. New and extensive simulations are going to be made considering different scenarios in order to derive the revenue potential, electricity storage potential and $\mathrm{CO}_{2}$ mitigation of the integrated steelworks with synthesis in future market situations.

Preliminary analyses using RAs parametrized with data from lab-scale reactors allowed to derive drivers but also potential obstacles of the concept. Especially, the development of the technical and economic dimension is of main importance for the long-term profitability: technologically, both the efficiency of the reactors and their expenses for start-up processes and load changes show a high sensitivity on the results. In particular, the start-up behaviour is a strong driver for the economic performance, as it defines the dynamic response of the reactors in volatile markets. Applying start-up expenses of the bench-scale reactors lead to very low hours of operation of the methanation ( $40 \mathrm{~h}$ per year), i.e., representing a negligible share of the total gas consumed in the steelworks. However, those expenses are hardly representative for a system in the MW-scale. Considering only $10 \%$ of the start-up costs 

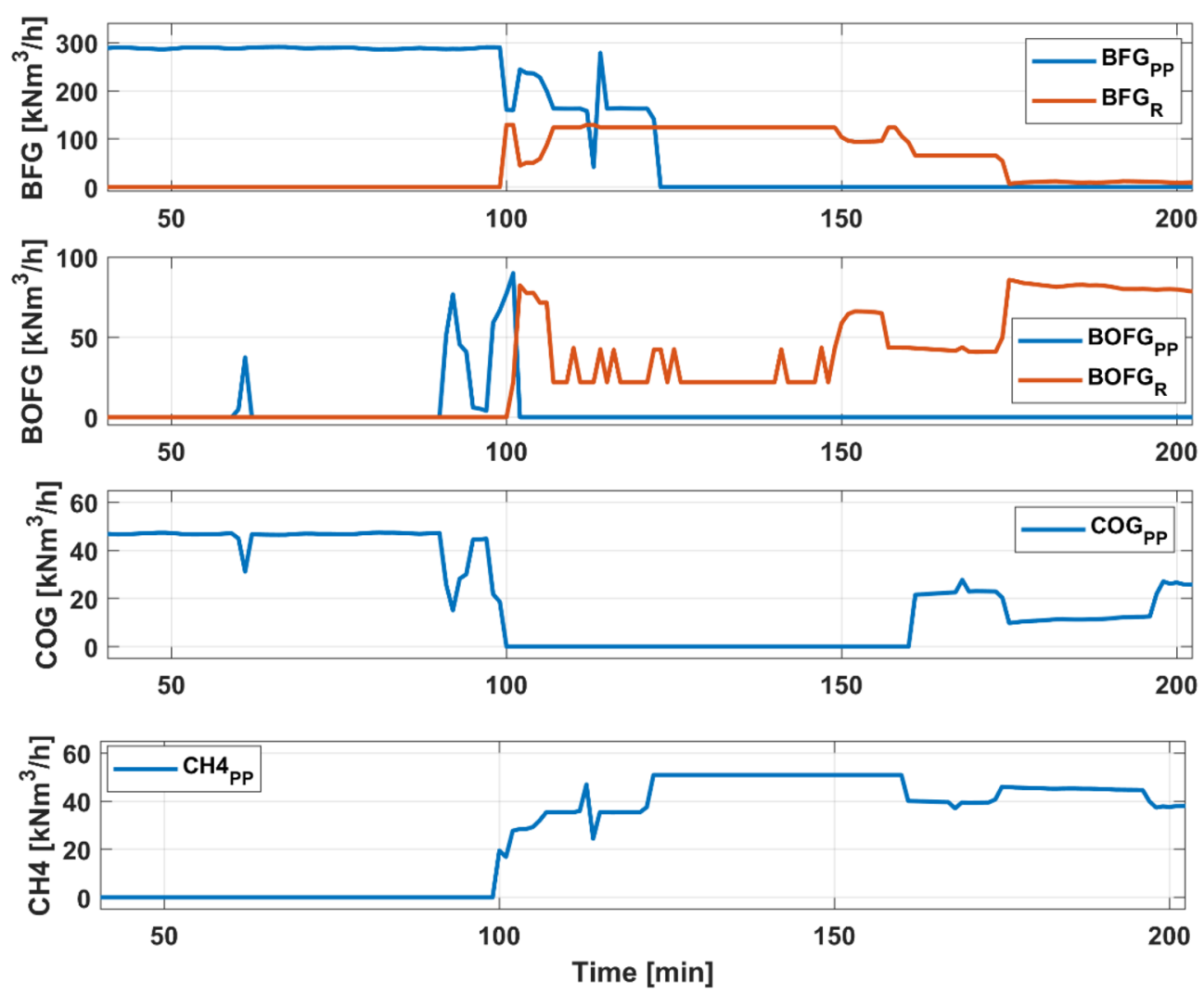

Fig. 7. Off-gases management through the dispatch controller agent.

increased the full load hours of the methanation by a factor of 5 . The methanation furthermore strongly benefits from an increase in electricity price volatility. Increasing price volatility by $50 \%$ improved the capacity utilization by a factor of 3 to 4 . Also, rising $\mathrm{CO}_{2}$ prices and a larger spread between natural gas and electricity prices enhanced the long-term profitability of the concept.

\section{Advantages and barriers of the application of agent-based solutions in steelworks}

Steel manufacturing industries operate in dynamic environments where markets are frequently shifting and the pressure of competition due to globalization is incredibly high. Moreover, new digital technologies are constantly emerging and their integration in the production chain, although potentially beneficial, is not always straightforward. As a consequence, steelworks are shifting to I4.0 paradigm to be more competitive through increasing efficiency and productivity, reducing costs and improving products quality. In this scenario, agent-based technologies provide an effective means to develop solutions to efficiently overcome such problems, by moving an important step ahead towards the concept of Smart Factory obtained by designing and implementing distributed smart manufacturing systems.

In Section 3, some applications of MASs have been presented to address some challenges of the steel sector. Agent-based solutions have been adopted to show their functionalities as well as their benefits through simulations in real contexts. Looking at the examples described in the
Section 3, the main advantages in using agent-based technologies in steelworks are the following:

- modelling and conceptualization of processes, resources, human operators through high-level languages and development tools, to facilitate monitoring, high level control, and optimization of production. The use of agent abstraction for modelling and simulating systems, also referred as Agent-Based Modelling and Simulation (ABMS), is a promising approach for simulating the behaviour of complex dynamic systems. OOP languages are commonly used for implementing agent development toolkits and frameworks, while simulation platforms such as AnyLogic(C) allow building agent-based models where the focus is directly on individual objects, their behaviour, and their interaction;

- describing steelworks by the interaction of its single agents. MASs also allow considering imperfections in decisionmaking and communication of established structures, enabling to simulate a close to real life behaviour;

- intelligent management (which is exemplarily applied to process off-gases in the second above-described use case) aimed at incrementing the economic and environmental sustainability of production;

- optimization of manufacturing process planning and scheduling through the efficient exploitation and allocation of plant resources. In the dynamic resource allocation example, the MAS builds a flexible production scheduling which maximizes the productivity by reducing waste of materials and times especially in perturbed scenarios; 
- modular approach, which allows easily and effectively varying the scenario and plant configuration to be investigated. In the first example, different perturbed scenarios can be considered to evaluate the possible capacity losses of the production, while in the second example, different strategies can be evaluated to improve the revenues while reducing $\mathrm{CO}_{2}$ emissions;

- robustness against dynamic changes and disturbances of the production. MASs through their distributed decision making promptly react and mitigate the disturbances effects as shown in the first example;

- enhance communication among subprocesses as well as cooperation among departments. In both examples, the different subprocess are interconnected through the communication capabilities of agents, which allow them cooperating for solving complex problems. For instance, the BSA interfaces with the volatile market and communicate with the DCA in order to handles off-gas management. The approach may also be used to interconnect different departments such as the Sales department and the Logistics department in order to coordinate the shipment of sold finished products;

- agents can be deployed to common IT infrastructures and wrap legacy software systems. As reported in the dynamic resource allocation example, the MAS was deployed through the well established paradigm of P2P taking into account the physical plant constraints.

However, there are still barriers, either technology- or human factor-related, that obstruct the adoption of agentbased solutions for industrial applications as reported in $[42,43]$. With respect to the steel sector, the main barriers according to the experience gained not only through the examples reported in the present paper can be the following:

- most of the optimization tasks in companies, e.g., logistics, production scheduling, etc. are usually carried out though centralized approaches, thus the controlcentric view is deep-rooted inside companies, which lack of confidence in decentralized and modular solutions and sometimes hamper their adoption;

- most IT systems implemented in the steelworks still rely on a ISA95-based pyramidal architecture, which does not help the full implementation of MASs (for instance, due to limited and mostly vertical data circulation). On the other hand, MASs benefit from the implementation of smart networks based on the principles of IIoT, providing fast access to data coming from different sources in the plant and possibly integrating Cloud and Edge Computing in view of breaking IT and Operational Technology (OT) barriers and enabling real time services;

- agent-based solutions and concepts may be hard to understand and their potential is either unknown or largely underestimated;

- skilled people, both workers and managers, and know how about new digital technologies are missing. This is due to lack of suitable educational programs and training before and after the introduction of new technologies, as well as investments in training and education. A relevant age gap is also foreseen between workers currently employed and future employees, which can create knowledge transfer issues and hamper the deployment of AI in the steel field.

\section{Conclusions}

In conclusion, the systematic use of agent-based technology in steel manufacturing industry offers several advantages in terms of quality, reliability, efficiency, robustness, autonomy, time, and costs in comparison to traditional manufacturing systems. In the context of Industry 4.0, steelworks are undergoing relevant transformation through the digitalization and thus the adoption of MASs seems very promising and represents a realistic solution for the set of steel industrial challenges. In this paper some applications of MASs have been presented to address some of the challenges showing their functionalities and benefits. Nevertheless, some barriers exist that need to be overcome for the full adoption of the agent-based technology in the steel sector.

The smooth introduction of MASs in steel production systems by testing and demonstrating the technology through simulation tools may be the solution to address concerns and doubts from industrial side. Furthermore, highlighting the improved performance obtained through MASs compared to traditional and adopted solutions may be a reason to convince steelworks companies about their potentials. Another aspect to consider is the costs in the development and maintenance of agent-based technologies. An assessment about costs and benefits of the proposed agent-based solutions may be useful for companies to understand the corresponding profit, which would derive from their investments. Finally, in order to speed up and facilitate MASs deployment, user-friendly tools and human-machine interface need to be developed, which hide the underlying complexity of the proposed solutions supported by detailed documentation and improve the level of acceptance from industrial side. These developments need to come together with suitable training and upskilling initiatives, which increase the knowledge and the confidence of the end-users toward these systems by helping them to appreciate benefits and potentials.

Acknowledgments. The work described in the present paper has been developed within the project entitled "Integrated and intelligent upgrade of carbon sources" (Ref. i3upgrade, Grant Agreement No. 800659) that has received funding from the Research Fund for Coal and Steel of the European Union, which is gratefully acknowledged. The sole responsibility of the issues treated in the present paper lies with the authors; the Commission is not responsible for any use that may be made of the information contained therein.

\section{References}

1. European Steel Association, European Steel in Figures 2020, 2020, Available from https://www.eurofer.eu/assets/Uploads/Europe an-Steel-in-Figures-2020.pdf [Online accessed: 6/10/2021] 
2. D. Ibarra, J. Ganzarain, I.J. Igartua, Business model innovation through Industry 4.0: A review, Proc. Manuf. 22, 4-10 (2018), https://doi.org/10.1016/j.promfg.2018.03.002

3. T.A. Branca, B. Fornai, V. Colla, et al., The challenge of digitalization in the steel sector, Metals 10(2), 1-23 (2020), https://doi.org/10.3390/met10020288

4. International Society of Automation, ANSI/ISA-95.00.01 Enterprise-Control System Integration-Part I: Models and Terminology, 2010

5. K. Schweichhart, Reference Architectural Model Industry 4.0 (RAMI 4.0), 2016, Available from https://ec.europa.eu/ futurium/en/system/files/ged/a2-schweichhart-referen ce_architectural_model_industrie_4.0_rami_4.0.pdf

6. V. Colla, C. Pietrosanti, E. Malfa, et al., Environment 4.0: How digitalization and machine learning can improve the environmental footprint of the steel production processes, Materiaux \& Techniques 108(5-6), (2020), https://doi.org/ $10.1051 / \mathrm{mattech} / 2021007$

7. T.A. Branca, V. Colla, D. Algermissen, et al., Reuse and recycling of by-products in the steel sector: Recent achievements paving the way to circular economy and industrial symbiosis in Europe, Metals 10(3), (2020), https://doi.org/ $10.3390 /$ met10030345

8. J. Feliks, K. Majewska, Agent-based modeling of steel production processes under uncertainty, in: Proceedings 24th International Conference on Metallurgy and Materials, METAL 2015, 2015, pp. 1739-1744

9. W. Shen, Q. Hao, H.J. Yoon, et al., Applications of agentbased systems in intelligent manufacturing: An updated review, Adv. Eng. Inform. 20, 415-431 (2006), https://doi. org $/ 10.1016 /$ j.aei.2006.05.004

10. S. Karnouskos, P. Leitao, L. Ribeiro, et al., Industrial agents as a key enabler for realizing industrial cyber-physical systems: Multiagent systems entering industry 4.0, IEEE Ind. Electron. Mag. 14(3), 18-32 (2020), https://doi.org/ 10.1109/MIE.2019.2962225

11. V. Gorodetsky, V. Larukchin, P. Skobelev, Conceptual model of digital platform for enterprises of industry 5.0, in: Studies in Computational Intelligence, 2020, pp. 35-40, https://doi.org/10.1007/978-3-030-32258-8_4

12. L. Monostori, J. Váncza, S.R.T. Kumara, Agent-based systems for manufacturing, CIRP Ann. Manuf. Technol. 55(2), 697720 (2006), https://doi.org/10.1016/j.cirp.2006.10.004

13. S. Jacobi, C. Madrigal-Mora, E. León-Soto, et al., AgentSteel: An agent-based online system for the planning and observation of steel production, in: Proceedings of the International Conference on Autonomous Agents, 2005, pp. $155-160$

14. L. Sun, F. Luan, A multi-agent framework for the scheduling of steel-making and continuous casting process with lagrangian relaxation neural networks, IFAC-PapersOnLine $\mathbf{2 8 ( 2 5 ) ,} \quad 108-113$ (2015), https://doi.org/10.1016/j.ifa col.2015.11.068

15. M.J. Neuer, F. Marchiori, A. Ebel, et al., Dynamic reallocation and rescheduling of steel products using agents with strategical anticipation and virtual market structures, IFAC-PapersOnLine 49(20), 232-237 (2016), https://doi. org $/ 10.1016 /$ j.ifacol.2016.10.126

16. F. Marchiori, A. Belloni, M. Benini, et al., Integrated dynamic energy management for steel production, Energy Proc. 105, 2772-2777 (2017), https://doi.org/10.1016/j. egypro.2017.03.597
17. F. Marchiori, M. Benini, S. Cateni, et al., Agent-based approach for energy demand-side management, Stahl und Eisen 138(2), 25-29 (2018)

18. S. Franklin, A. Graesser, Is it an agent, or just a program? A taxomony of autonomous agents, in: J.P. Müller, M.J. Wooldridge, N.R. Jennings, eds., Intelligent Agents III Agent Theories, Architectures, and Languages, Springer Berlin Heidelberg, 1996, pp. 21-35

19. Y. Labrou, T. Finin, Y. Peng, Agent communication languages: The current landscape, IEEE Intell. Syst. Their Appl. 14(2), 45-52 (1999), https://doi.org/10.1109/ 5254.757631

20. H.S. Nwana, L. Lee, N.R. Jennings, Co-ordination in software agent systems, BT Technol. J., 42-58 (1996)

21. J.E. Doran, S. Franklin, N.R. Jennings, et al., On cooperation in multi-agent systems, Knowl. Eng. Rev., 1-7 (1997), https://doi.org/10.1017/S0269888997003111

22. T. Labeodan, K. Aduda, G. Boxem, et al., On the application of multi-agent systems in buildings for improved building operations, performance and smart grid interaction - A survey, Renew. Sustain. Energy Rev. 50, 1405-1414 (2015), Elsevier Ltd, https://doi.org/10.1016/j.rser.2015.05.081

23. G.H. Merabet, M. Essaaidi, H. Talei, et al., Applications of multi-agent systems in smart grids: A survey, in: Proceedings on International Conference on Multimedia Computing and Systems, Sep. 2014, pp. 1088-1094, https://doi.org/10.1109/ ICMCS.2014.6911384

24. B. Chen, H.H. Cheng, A review of the applications of agent technology in traffic and transportation systems, IEEE Trans. Intell. Transp. Syst. 11, 485-497 (2010), https://doi. org/10.1109/TITS.2010.2048313

25. E. Shakshuki, M. Reid, Multi-agent system applications in healthcare: Current technology and future roadmap, Proc. Comput. Sci. 52(1), 252-261 (2015), https://doi.org/ 10.1016/j.procs.2015.05.071

26. V. Iannino, V. Colla, J. Denker, et al., A CPS-based simulation platform for long production factories, Metals $\mathbf{9}$ (10), 1-20 (2019), https://doi.org/10.3390/met9101025

27. V. Iannino, C. Mocci, M. Vannocci, et al., An event-driven agent-based simulation model for industrial processes, Appl. Sci. 10(12), 1-22 (2020), https://doi.org/10.3390/ app10124343

28. L. Tang, J. Liu, A. Rong, et al., A review of planning and scheduling systems and methods for integrated steel production, Eur. J. Oper. Res. 133(1), 1-20 (2001), https://doi.org/10.1016/S0377-2217(00)00240-X

29. M. Iglesias-Escudero, J. Villanueva-Balsera, F. OrtegaFernandez, et al., Planning and scheduling with uncertainty in the steel sector: A review, Appl. Sci. (2019), https://doi. org/10.3390/app9132692

30. J. Zhao, Q.L. Liu, W. Wang, Models and algorithms of production scheduling in tandem cold rolling, Zidonghua Xuebao/Acta Autom. Sin. 34(5), 565-573 (2008), https:// doi.org/10.3724/SP.J.1004.2008.00565

31. J. Wan, et al., Toward dynamic resources management for IoT-based manufacturing, IEEE Commun. Mag. 56(2), 5259 (2018), https://doi.org/10.1109/MCOM.2018.1700629

32. V. Iannino, C. Mocci, V. Colla, A brokering-based interaction protocol for dynamic resource allocation in steel production processes, in: Advances in Intelligent Systems and Computing, 1368 AISC, pp. 119-129, 2021, https://doi.org/10.1007/ 978-3-030-72654-6 12 
33. V. Iannino, C. Mocci, V. Colla, A hybrid peer-to-peer architecture for agent-based steel manufacturing processes, IFAC-PapersOnLine 54(1), 528-33 (2021), https://doi.org/ 10.1016/j.ifacol.2021.08.167

34. O. Taisir, Total productive maintenance review and overall equipment effectiveness measurement, Jordan J. Mech. Ind. Eng. 4(4), 517-522 (2010)

35. S. Kumar, R. Bhushan, S. Swaroop, Study of total productive maintenance \& its implementation approach in steel manufacturing industry: A case study of equipment wise breakdown analysis, Int. Res. J. Eng. Technol. 4(8), 608-613 (2017), Available from https://www.academia.edu/ 34509469/Study of total productive maintenance an d its implementation approach in steel manufacturin g_industry_A_case_study_of_equipment_wise_break down_analysis [Online accessed: 6/16/2021]

36. A. Hauser, M. Weitzer, S. Gunsch, et al., Dynamic hydrogenintensified methanation of synthetic by-product gases from steelworks, Fuel Process. Technol. 217, 1-10 (2021), https:/ doi.org/10.1016/j.fuproc.2020.106701

37. A. Zaccara, A. Petrucciani, I. Matino, et al., Renewable hydrogen production processes for the off-gas valorization in integrated steelworks through hydrogen intensified methane and methanol syntheses, Metals 10(11), 1-24 (2020), https://doi.org/10.3390/met10111535
38. M. Bampaou, K. Panopoulos, P. Seferlis, et al., Integration of renewable hydrogen production in steelworks off-gases for the synthesis of methanol and methane, Energies 14(10), 2904 (2021), https://doi.org/10.3390/en14102904

39. I. Matino, S. Dettori, V. Colla, et al., Forecasting blast furnace gas production and demand through echo state neural network-based models: Pave the way to off-gas optimized management, Appl. Energy 253, (2019), https:// doi.org/10.1016/j.apenergy.2019.113578

40. S. Dettori, I. Matino, V. Colla, et al., A deep learning-based approach for forecasting off-gas production and consumption in the blast furnace, Neutr. Comput. Appl., 1-14 (2021), https://doi.org/10.1007/s00521-021-05984-x

41. S. Dettori, A. Maddaloni, F. Galli, et al., Steam turbine rotor stress control through nonlinear model predictive control, Energies 14(13), 1-30 (2021), https://doi.org/10.3390/ en14133998

42. P. Leitão, V. Mařík, P. Vrba, Past, present, and future of industrial agent applications, IEEE Trans. Ind. Inform.

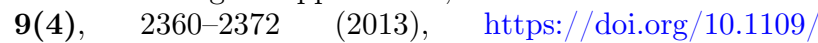
TII.2012.2222034

43. V. Marik, V. Gorodetsky, P. Skobelev, Multi-agent technology for industrial applications: Barriers and trends, IEEE Trans. Syst. Man, Cybern.: Syst. 1980-1987 (2020), https:// doi.org/10.1109/SMC42975.2020.9283071

Cite this article as: Vincenzo Iannino, Valentina Colla, Claudio Mocci, Ismael Matino, Stefano Dettori, Sebastian Kolb, Thomas Plankenbühler, Jürgen Karl, Multi-agent systems to improve efficiency in steelworks, Matériaux \& Techniques 109, 502 (2021) 